\title{
What is localized neuropathic pain? A first proposal to characterize and define a widely used term
}

Gérard Mick*, Ralf Baron', Nanna Brix Finnerup², Guy Hans³, Kai-Uwe Kern", Birgit Brett ${ }^{5} \&$ Robert H Dworkin ${ }^{6}$
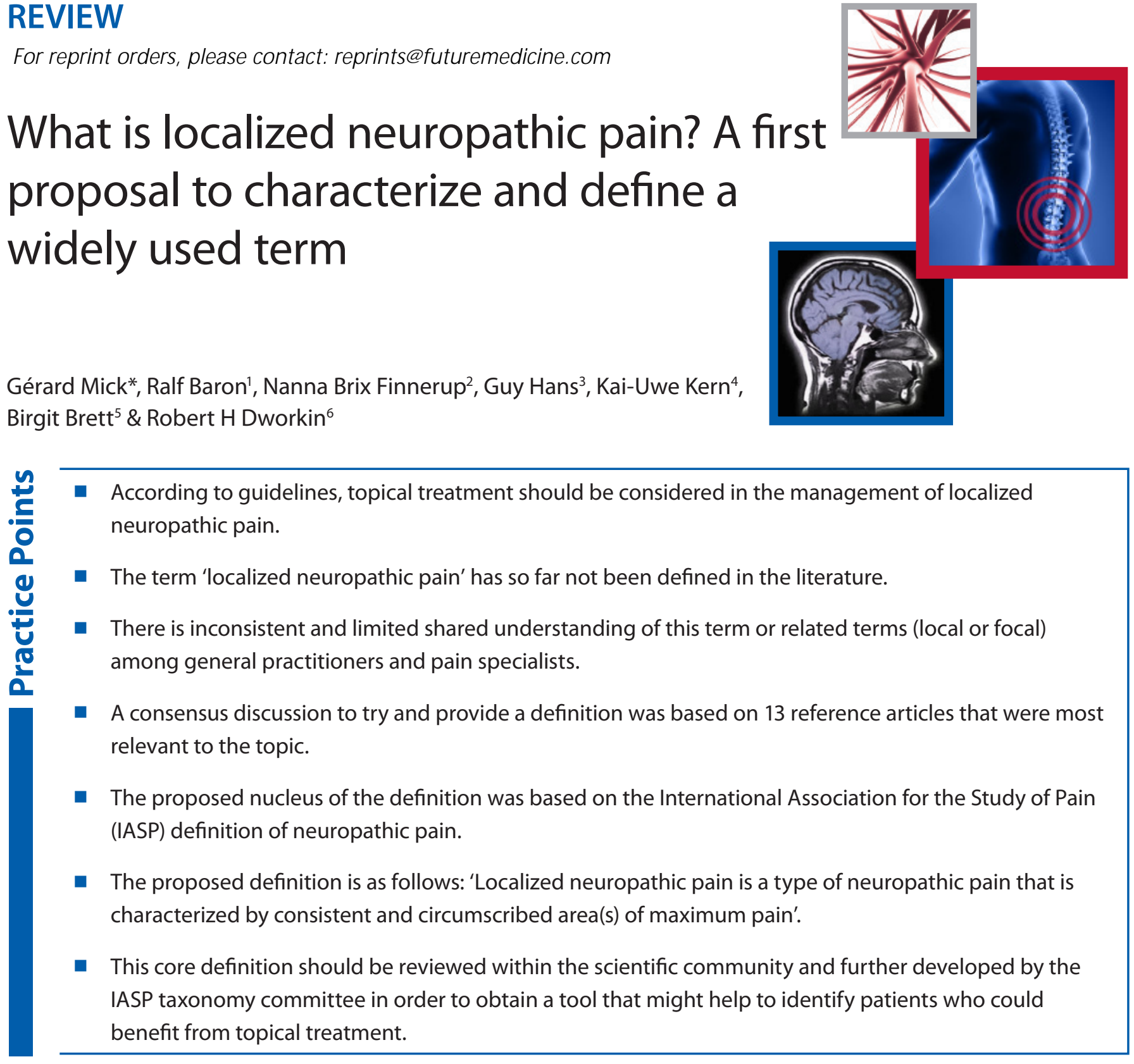

SUMMARY According to several guidelines, topical agents should be considered for the pharmacological management of localized neuropathic pain. As a definition for the term 'localized neuropathic pain' that might facilitate easier identification of patients who are putatively responsive to topical treatments could not be found in the literature, six pain specialists met in 2010 to address this challenging issue. The following nucleus of a definition that is based on the International Association for the Study of Pain (IASP) definition of neuropathic pain, is the most detailed that can currently be proposed: 'Localized neuropathic pain is a type of neuropathic pain that is characterized by consistent and circumscribed

'Division of Neurological Pain Research \& Therapy, Department of Neurology, University Hospital Schleswig-Holstein, Kiel, Germany

2Danish Pain Research Centre, Aarhus University Hospital, Aarhus, Denmark

${ }^{3}$ Multidisciplinary Pain Center, Antwerp University Hospital, Edegem, Belgium

${ }^{4}$ Center for Pain Medicine \& Palliative Care, Wiesbaden, Germany

${ }^{5}$ Brett Medical Writing, Pulheim, Germany

${ }^{6}$ Departments of Anesthesiology \& Neurology, University of Rochester School of Medicine \& Dentistry, Rochester, NY, USA

*Author for correspondence: Center for Pain Evaluation \& Treatment, University Neurological Hospital, BP Lyon Montchat, 69384 Lyon

Cedex 3, France; Tel.: +3368013 40 09; Fax: +33 47667 15 43; gerard.mick@ch-voiron.fr

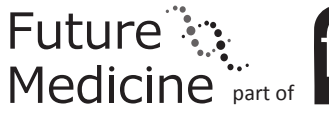


area(s) of maximum pain'. An extended version of this core definition and the difficulties in covering all aspects of localized neuropathic pain are presented, and discussions within the scientific community are encouraged to develop a definition that might help to identify patients who could benefit most from topical treatment.

Neuropathic pain was originally defined by the International Association for the Study of Pain (IASP) as 'pain initiated or caused by a primary lesion or dysfunction in the nervous system', and more recently as 'pain arising as a direct consequence of a lesion or disease affecting the somatosensory system' [1]. This revised definition has now been accepted by the IASP and updated in the taxonomy listing [101]. However, discussions in the scientific community are still ongoing [2]. Whereas nociceptive pain occurs as a result of tissue damage or disease in the presence of a functionally intact nervous system, neuropathic pain arises when the nervous system itself is damaged [3]. Despite recent progress in the understanding, diagnosis, pathophysiological mechanisms and treatment of neuropathic pain [4-11], many patients remain refractory to, or intolerant of, existing pharmacological treatments. The localization of neuropathic pain varies among patients, presenting as widely distributed (e.g., hemibody in post-stroke central pain) in some, or in a relatively restricted distribution (e.g., distal part of a dermatoma in postherpetic neuralgia $[\mathrm{PHN}])$ in others.

Topically applied agents with different mechanisms of action, such as capsaicin, lidocaine and NSAIDs, have been used for the localized treatment of peripheral neuropathic pain in clinical and experimental settings [12]. Although topically applied agents are widely used for peripheral neuropathic pain, existing guidelines and treatment recommendations $[4-6,13,14]$ and reviews on topical treatments $[12,15]$ do not describe the clinical characteristics of patients for whom a topical approach should be recommended. In considering which patients with neuropathic pain are candidates for topical treatments in clinical settings, terms such as 'localized', 'focal' or 'discrete' have been used to describe the distribution of their pain in the case of peripheral neuropathic pain, or reference was made to the underlying pain diagnosis for which the treatment is indicated. The majority of randomized clinical trials in neuropathic pain have investigated patients within a specific etiology, such as PHN or painful diabetic peripheral neuropathy (DPN) $[4,8]$. Although most clinicians and researchers would agree that, for example, peripheral neuropathic pain such as PHN or postsurgical scar pain are localized, with an area of pain usually related to the primary lesion, it has been less clear whether the term 'localized' applies to pain in patients with other types of peripheral neuropathic pain, such as painful DPN or other polyneuropathies with a distal distribution.

The treatment algorithms for different types of chronic pain vary because of the different pathophysiologic mechanisms of pain and the different mechanisms of action of available treatments. Hence, clinicians evaluating patients with chronic pain must determine whether the patient suffers from a nociceptive, neuropathic or another pain condition (e.g., fibromyalgia); if neuropathic pain, whether the initiating lesion is peripheral or central; and if peripheral neuropathic pain, whether the pain is widely distributed or relatively localized. Such assessment might make evidence-based treatment possible [3]. For patients with peripheral neuropathic pain, the treatment can include systemic agents or topically applied agents or their combination. However, systemic and topical treatments for neuropathic pain have different profiles of benefits and risks, with topical treatments associated with relatively few systemic side effects and drug-drug interactions. Moreover, the efficacy of topical treatments has been demonstrated in randomized trials that only enrolled patients with relatively localized pain conditions. For these reasons, a definition of 'localized neuropathic pain' (LNP), for which a topical treatment should be considered, would facilitate an evidence-based treatment approach to neuropathic pain. In order to propose a definition of LNP for daily clinical practice, the authors of this article (six of them experts on neuropathic pain) met in 2010 and reviewed the relevant literature, evidence and clinical considerations. This article presents a summary of this consensus meeting and subsequent discussions.

\section{Literature search}

An Embase and a PubMed literature search using the keywords 'peripheral', 'local', 'focal' and 'localized/localised' in combination with 
'neuropathic pain' was conducted in February 2010 to find publications with a definition for LNP. A total of 1211 English language publications or publications with an English abstract (human subjects) were retrieved. These were further screened for publications using the term 'localized neuropathic pain' (108 references), 'defined as' (37 references) and 'definition' (16 references). In addition, citations used in the retrieved literature and present in the literature pertaining to the definition and diagnosis of neuropathic pain $[3,16,101]$ and in the guidelines, recommendations and treatment algorithms for neuropathic pain $[4,6]$ and reviews on topical treatments for neuropathic pain $[12,15]$ were screened for a definition of 'localized', 'local' or 'focal neuropathic pain'. No article, however, provided a definition, although these terms were used. Finally, as a basis for the consensus discussion, 13 reference articles that came closest to the topic were selected $[3,4,11,15-23,101]$.

\section{Understanding of the notion of LNP among physicians}

Considering the lack of a definition in the literature, it is not surprising that there is inconsistent and limited shared understanding of this term or related terms (local or focal) among physicians. This was clearly shown by face-to-face interviews (average duration $45 \mathrm{~min}$ ) with 585 general practitioners and 284 pain specialists conducted in several western European countries from December 2008 to January 2010. The survey was performed by the market research company GfK HealthCare, Germany, using their panel of physicians with a record of regularly treating patients with pain (at least 3 years of experience in pain treatment). A randomized sample was taken from this pool of physicians. The questionnaire comprised 58 questions relating to neuropathic pain and treatment options. It consisted mainly of closed questions but included some explorative ones. A variety of answers were collected to the open question 'How would you define LNP?' [Grünenthal GmbH, Data on File]. The responses included very different items such as 'pain experienced at a particular location on a specific part of the body', 'burning pain', 'one specific nerve affected', 'pain resulting from nerve damage', 'precise delimitation of the painful area', 'intense pain' or 'local/easily localized pain'. Moreover, in some countries, as many as $70 \%$ of general practitioners and pain specialists provided 'I don't know' as an answer.

\section{Putative prevalence of LNP}

The results of the literature search were further screened for 'prevalence' and 72 publications were identified. On the basis of cross-sectional surveys, the prevalence of chronic pain with neuropathic pain characteristics was estimated as $6.9 \%$ in France [24], and prevalence of pain with a predominantly neuropathic origin was estimated as $8 \%$ in the UK [25]. A Dutch survey reported an annual incidence of neuropathic pain in almost $1 \%$ of the general population [26]. A recent review summarizes the available information on the prevalence of neuropathic pain [27]. However, generally, prevalence data are scarce and none of these publications contain estimates for the prevalence of LNP. To obtain preliminary estimates of the prevalence of LNP, the general practitioners and pain specialists who participated in the unpublished survey described in the previous section were asked about: the underlying neuropathic pain diagnosis their patients presented with (some answer choices such as PHN, DPN, neuropathic back pain and others were provided, but physicians could also list other neuropathic pain conditions); and how many of these patients suffered from LNP. For this purpose and in the absence of any approved official definition, the following definition of LNP was provided: 'Localized neuropathic pain is confined to a specific area not larger than a letter-size piece of paper. The area can be precisely isolated by tracing around the contours of it with a marking pen, for example'. Averaging across all neuropathic pain conditions, physicians reported that approximately $60 \%$ of patients had LNP (Figure 1).

\section{Towards a definition of LNP}

To develop a proposed consensus definition of LNP, various wordings that the participants believed to be potentially relevant to such a definition were discussed. These descriptive terms and phrases included 'localized', 'superficial', 'circumscribed', 'well-defined area of pain', 'abnormal skin sensation (decreased or increased sensation)', 'local', 'cutaneous', 'being able to point to the area of pain', 'area of significant pain', 'constant over time', 'unilateral' and 'bilateral'. Following discussion of these terms and phrases and their relevance to provide a proposed definition of LNP that could be used in evidence-based treatment, various definitions were proposed that 


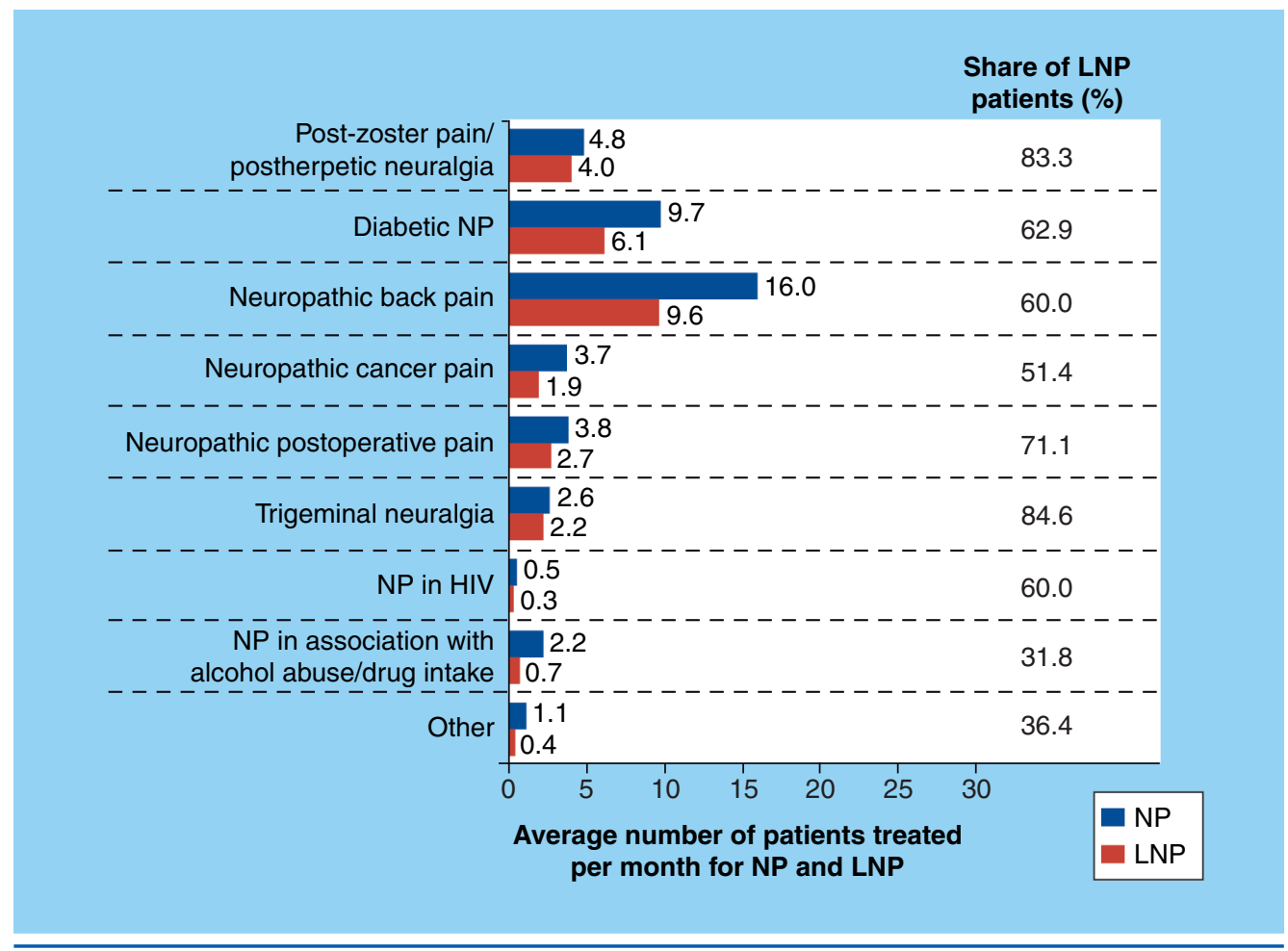

Figure 1. Putative prevalence of 'localized neuropathic pain' in patients $(n=869)$ attending general practitioners and pain specialists in western Europe.

LNP: Localized neuropathic pain; NP: Neuropathic pain.

considered LNP as neuropathic pain that: is localized to well-defined areas in which are found abnormally sensitive skin and/or spontaneous symptoms such as burning; is accompanied by abnormally sensitive skin and with consistent and circumscribed areas of significant pain; or is felt by the patient as significant in a circumscribed area in which the skin is abnormally sensitive. A consensus was finally reached regarding the following initial nucleus for a definition: 'Localized neuropathic pain is a type of neuropathic pain that is characterized by consistent and circumscribed area(s) of maximum pain'.

\section{Discussion}

A classification based on mechanisms and signs and symptoms, in addition to the traditional classification based on disease entities, anatomical location or histological observations, could be of more use for treatment selection in the future [28]. Although this holds promise, and there has been a lot of progress in this field, research has not yet reached the point at which one can clearly select a treatment on the basis of symptom profiles alone [9]. We believe that a definition of LNP could potentially contribute to advancement in treatment, as it is based on localized signs and symptoms.

As suggested in most neuropathic pain treatment guidelines, topically applied agents are among the better-tolerated treatments and are typically indicated for patients with LNP $[4,5]$. A definition of LNP is thus needed to clinically identify patients who are candidates for such treatments. The present work proposes a first nucleus for a definition for LNP, a type of neuropathic pain characterized by a circumscribed and consistent area of maximum pain. This definition is the most detailed that can be proposed, based on currently available evidences and literature.

Since the definition of neuropathic pain is assumed, this core definition for LNP would serve to describe what LNP is. However, the authors felt that in order to be useful for evidence-based treatment, the definition ought to be extended and the following wording is suggested: 'Localized neuropathic pain is a type of neuropathic pain that is characterized by consistent and circumscribed area(s) of maximum pain associated with negative or positive 
sensory signs and/or spontaneous symptoms characteristic of neuropathic pain'.

In peripheral neuropathic pain, there is almost always an area of abnormal sensation, and the patient's maximum pain is usually coexistent with or lies within an area of sensory alterations [3]. Thus, the proposed wording includes 'area(s) of maximum pain associated with negative or positive sensory signs'. It was further agreed that patients with LNP should be able to point to the location(s) of their pain or abnormally sensitive skin, in order to distinguish LNP from widely distributed or even widespread pain, and that this area of pain should remain constant over time.

Defining LNP proved to be more difficult than anticipated because of the diverse presentations and symptoms considered for inclusion in the definition. Whereas there was little ambiguity that LNP is often observed in certain pain conditions in which the area of pain is closely related to the origin of pain (e.g., postsurgical pain or PHN), LNP was more difficult to define for other conditions such as DPN or neuropathic low back pain. Patients with DPN may present with several distinct areas of localized pain, and neuropathic low back pain frequently includes nociceptive as well as neuropathic components ('mixed pain').

It was recognized that the proposed definition has several shortcomings. However, with the evidence available, it appears to be the most detailed definition that can be currently proposed. Moreover, there is to date no clinical predictor(s) for the patient response to a topical treatment. Thus, issues that need to be studied for improvement of the proposed core definition/ extended version and evaluation of its usefulness in therapeutic choice include:

- Do the terms 'consistent' and 'maximum' describe the only area(s) of pain that is significant for the patient and thus the optimal area for the application of a topical treatment?

- What is the upper limit for the size of LNP (i.e., how large can the area of pain and the area covered by a topical treatment be for a positive effect)?

- Will both superficial and deep pains respond to topical treatments?

- What is the relationship between response to treatment and the extent of deafferentation versus preservation of small or large afferent fibers in the area of pain?
- Are there potential exclusions for the proposed definition?

However, even the extended definition may not have the potential to facilitate evidencebased treatment of LNP, and does not provide an upper size limit for the area of significant pain for the patient and for the target area of topical agents. As LNP refers to the distribution of neuropathic pain, it should also be emphasized that topical treatments are usually considered for peripheral LNP and not for localized central pain conditions. This consensus statement represents a first step towards a clinical description of anatomical subtypes of neuropathic pain, and the authors propose that the taxonomy committee of the IASP could further develop this definition. Readers are also encouraged to contribute to the discussion. As a future perspective, clinical trials will be needed in order to validate an agreed definition of LNP and to examine whether this definition identifies patients who are most likely to respond to topical treatments.

\section{Future perspective}

Many patients with neuropathic pain, in particular the elderly, (professional) drivers or people who work with machinery might benefit from topical rather than systemic treatment, and quite a few new topical compounds are expected to become available in the coming years. A definition of LNP would ideally serve to identify patients who are most likely to respond to topical treatments.

This definition may contribute to a better and faster selection of treatment for LNP, thereby shortening the trial-and-error period that usually precedes the successful treatment of any neuropathic pain. This in turn may lead to faster pain relief, fewer systemic side effects and a better quality of life for the patient.

Acknowledgements

The consensus meeting on which this article is based was sponsored by Grünenthal GmbH, Germany.

Financial \& competing interests disclosure $G$ Mick has received in the past 3 years support for clinical research and educational programs from Grünenthal and Menarini, and compensation for consulting from AFSSAPS, Boehringer-Ingelheim, Grünenthal, JanssenCilag, Lilly, Menarini, Mundipharma, Pfizer and Sanof-Pasteur-MSD. 
$R$ Baron has received in the past 3 years honoraria from Allergan, Pfizer, Grünenthal, Medtronic, Mundipharma, Eisai, Sanof-Pasteur, UCB Pharma, Lilly, Astellas, Böhringer-Ingelheim and Genzyme and received research funding from Pfizer, Grünenthal and Genzyme.

NB Finnerup has received in the past 3 years research support and honoraria from Grünenthal.

$G$ Hans has received in the past 3 years research funding from Grünenthal, as well as consultancy honoraria from Grünenthal.

$K$-U Kern has received in the past 3 years consultancy honoraria from Astellas, Berlin Chemie, Eisai, Grünenthal, Lilly and Medi Bayreuth.

$B$ Brett has received in the past 3 years honoraria for writing and editorial assistance, publication coordination and translation services from Bayer Health Care, Grünenthal, Janssen-Cilag, Merz Pharmaceuticals and UCB Pharma.
$R H$ Dworkin has received in the past 3 years research support from the Montel Williams Foundation, Serono, US FDA and US NIH, and compensation for consulting from Acura, Adynxx, Allergan, Analgesic Solutions, Astellas, Avanir, Bayer, Biogen, Cardiome, Dainippon Sumitomo, Depomed, Endo, Epicept, Flexion, Glenmark, Grünenthal, Infinity, Inhibitex, Jazz, Johnson \& Johnson, King, Lilly, McNeil, MediciNova, Merck, MMS Holdings, NeurogesX, Ono, Seikagaku, Smith \& Nephew, Spinifex, Pfizer, Phillips Respironics, RCT Logic, Sanofi-Aventis, Theravance and the US Department of Veterans Affairs.

The authors have no other relevant affiliations or financial involvement with any organization or entity with a financial interest in or financial conflict with the subject matter or materials discussed in the manuscript apart from those disclosed.

Writing assistance was utilized in the production of this manuscript. B Brett received honoraia from Grünenthal for providing writing and editorial assistence.

\section{References}

Papers of special note have been highlighted as:

- of interest

-. of considerable interest

1 Treede RD, Jensen TS, Campbell JN et al. Neuropathic pain: redefinition and a grading system for clinical and research purposes. Neurology 70, 1630-1635 (2008).

- Recent redefinition of neuropathic pain by an international expert panel, which is still being discussed by the scientific community. It also introduces a grading system of definite, probable or possible neuropathic pain.

2 Lynch ME, Clark AJ, Moulin DE, Watson CP. Modifications are suggested for the Special Interest Group (SIG) on Neuropathic Pain proposed definition and guidelines for neuropathic pain. Pain 152, 1682; author reply 1683-1684 (2011).

3 Haanpää M, Backonja MM, Bennett $M$ et al. Assessment of neuropathic pain in primary care. Am. J. Med. 122(Suppl. 10), S13-S21 (2009).

- Review providing comprehensive guidance to primary care physicians for understanding and identifying the neuropathic contribution to pain.

4 Dworkin RH, O'Connor AB, Backonja M et al. Pharmacologic management of neuropathic pain: evidence-based recommendations. Pain 132, 237-251 (2007).

- The Neuropathic Pain Special Interest Group (NeuPSIG) of the International
Association for the Study of Pain (IASP) guidelines.

5 Moulin DE, Clark AJ, Gilron I et al. Pharmacological management of chronic neuropathic pain: consensus statement and guidelines from the Canadian Pain Society. Pain Res. Manag. 12, 13-21 (2007).

-1 The Canadian Pain Society guidelines.

6 Attal N, Cruccu G, Baron R et al. EFNS guidelines on the pharmacological treatment of neuropathic pain: 2010 revision. Eur. J. Neurol. 17, 1113-E88 (2010).

-. The European Federation of Neurological Societies (EFNS) guidelines.

7 O'Connor AB, Dworkin RH. Treatment of neuropathic pain: an overview of recent guidelines. Am. J. Med. 122(10A), S22-S32 (2009).

- Comparison of the NeuPSIG, Canadian Pain Society and EFNS treatment guidelines for neuropathic pain.

8 Finnerup NB, Sindrup SH, Jensen TS. The evidence for pharmacological treatment of neuropathic pain. Pain 150, 573-581 (2010).

9 Baron R, Binder A, Wasner G. Neuropathic pain: diagnosis, pathophysiological mechanisms, and treatment. Lancet Neurol. 9, 807-819 (2010).

- Recent review providing the current state of knowledge concerning the mechanisms of neuropathic pain generation and the assessment and treatment of neuropathic pain. Although one topical agent is recommended for localized peripheral neuropathic pain in general, no clear definition is given.

10 Maier C. Baron R, Tölle TR et al. Quantitative sensory testing in the German Research Network on Neuropathic Pain (DFNS): somatosensory abnormalities in 1236 patients with different neuropathic pain syndromes. Pain 150, 439-450 (2010).

11 Haanpää M, Attal N, Backonja M et al. NeuPSIG guidelines on neuropathic pain assessment. Pain 152(1), 14-27 (2011)

12 Sawynok J. Topical and peripherally acting analgesics. Pharmacol. Rev. 55, 1-20 (2003).

- Comprehensive review of topical compounds (available and under investigation) and the rationale for using topicals for the treatment of localized neuropathic pain.

13 Finnerup NB, Otto M, McQuay HJ, Jensen TS, Sindrup SH. Algorithm for neuropathic pain treatment: an evidence based proposal. Pain 118, 289-305 (2005).

14 Dworkin RH, O'Connor AB, Audette J et al. Recommendations for the pharmacological management of neuropathic pain: an overview and literature update. Mayo Clin. Proc. 85 (Suppl. 3), S3-S14 (2010).

-1 Review of the evidence-based guidelines developed by the NeuPSIG of the IASP, taking into consideration recent clinical trials that were not available at the time the guidelines were published.

15 de Leon-Casasola OA. Multimodal approaches to the management of neuropathic pain: the role of topical analgesia. J. Pain Symptom Manage. 33, 356-364 (2007). 
- Many varied topical analgesics for neuropathic pain are reviewed, with special emphasis on the molecular basis of pain generation and analgesia.

16 Geber C, Baumgärtner U, Schwab R et al. Revised definition of neuropathic pain and its grading system: an open case series illustrating its use in clinical practice. Am. J. Med. 122 (Suppl. 10), S3-S12 (2009).

- Open case series demonstrating how the grading system suggested by Treede et al. [1] can be applied for the diagnosis of neuropathic pain conditions in clinical practice.

17 Meier T, Wasner G, Faust M et al. Efficacy of lidocaine patch $5 \%$ in the treatment of focal peripheral neuropathic pain syndromes: a randomized, double-blind, placebocontrolled study. Pain 106, 151-158 (2003).

18 Sawynok J. Recent findings surrounding topical antidepressants as analgesics and review of existing and emerging topical analgesics. Adv. Stud. Med. 3, S635-S641 (2003).

19 Nayak S, Cunliffe M. Lidocaine 5\% patch for localized chronic neuropathic pain in adolescents: report of five cases. Paediatr. Anaesth. 18, 554-558 (2008).

20 Cruz-Almeida Y, Felix ER, Martinez-Arizala A, Widerstrom-Noga EG. Pain symptom profiles in persons with spinal cord injury. Pain Med. 10, 1246-1259 (2009).

21 Derry S, Lloyd R, Moore RA, McQuay HJ. Topical capsaicin for chronic neuropathic pain in adults. Cochrane Database Syst. Rev. 4, CD007393 (2009).

22 Schestatsky P, Nascimento OJ. What do general neurologists need to know about neuropathic pain? Arq. Neuropsiquiatr. 67, 741-749 (2009).

23 Paster Z, Morris CM. Treatment of the localized pain of postherpetic neuralgia. Postgrad. Med. 122, 91-107 (2010).

24 Bouhassira D, Lanteri-Minet M, Attal N, Laurent B, Touboul C. Prevalence of chronic pain with neuropathic characteristics in the general population. Pain 136, 380-387 (2008).

25 Torrance N, Smith BH, Bennett MI, Lee AJ. The epidemiology of chronic pain of predominantly neuropathic origin: results from a general population survey. J. Pain. 7, 281-289 (2006).
26 Dieleman JP, Kerklaan J, Huygen FJ, Bouma PA, Sturkenboom MC. Incidence rates and treatment of neuropathic pain conditions in the general population. Pain 137, 681-688 (2008).

27 Smith BH, Torrance N. Epidemiology of neuropathic pain. Pain Manage. 1, 87-96 (2011).

28 Baron R, Tölle TR. Assessment and diagnosis of neuropathic pain. Curr. Opin. Support. Palliat. Care 2, 1-8 (2008).

\section{- Website}

101 IASP Task Force on Taxonomy. Part III: Pain Terms, A Current List with Definitions and Notes on Usage Classification of Chronic Pain (2nd Edition). Merskey H, Bogduk N (Eds). IASP Press, Seattle, USA, 209-214 (1994). www.iasp-pain.org/AM/Template. cfm?Section=Pain_Defi...isplay.cfm \& Conte $\mathrm{ntID}=1728$ \# Neuropathicpain

- Current official IASP definition of neuropathic pain; the IASP has now updated the taxonomy listing and accepted the redefinition by Treede et al. [1] . 